\title{
SINDROME DE WYBURN MASON, UMA FACOMATOSE MISTA
}

\author{
Luís Renato Mello*
}

A angiomatose facio-mesodiencefálica, conhecida na literatura anglo-saxã como sindrome de Wyburn Mason foi referida pela primeira vez por Bonnet, Dechaume e Blanc em $1936^{1}$. Em 1942, Wyburn Mason ${ }^{16}$ publicou um estudo detalhado de todos os casos encontrados na literatura onde tivessem sido observados angiomas faciais, retinianos e mesodiencefálicos. Somente 10 anos após, Fishgold e col. ${ }^{5}$ estudaram os aspectos radiológicos em um caso. A revisão bibliográfica que realizamos mostrou tratar-se de entidade rara, uma vez que encontramos 31 casos publicados com este diagnóstico, sendo que destes somente 11 apresentavam estudo angiográfico, assim mesmo muitas vezes inadequado ou incompleto. Os restantes foram comprovados anátomo-patologicamente.

Trata-se de uma displasia vascular congênita originada por um defeito mesoectodérmico que ocorreria na quarta fase embriológica de Streeter, onde há cruzamentos e rearranjo dos plexos embrionários pré-capilares venosos e arteriais. Nesta fase a separação de ambos é por apenas a espessura de dois endotélios ${ }^{4}, 11$.

Como substrato anátomo-patológico apresenta uma triade de angiomas na face, na retina e no mesodiencéfalo. O angioma de face é geralmente plano ou hipertrófico, de cor violácea ou vinhosa. A lesão retiniana varia mais, aparecendo desde uma comunicação entre uma artéria e uma veia dilatada, até um aumento do número e engurgitamento dos vasos retinianos, nunca sendo pulsátil. A malformação cerebral caracteriza-se pela presença de angiomas artério-venosos geralmente unilaterais ocupando as regiôes diencefálicas e mesencefálicas, estendendo-se por vezes desde o quisma e tracto óptico, até o diencéfalo e córtex occipital e, mais raramente, à fossa posterior. Em dois casos a necrópsia mostrou angiomas bilaterais ${ }^{13,16}$. A triade nem sempre é completa, tendo sido descritos dois casos sem lesões retinianas ${ }^{2}$.

A sintomatologia aparece geralmente na $2^{a}$ e $3^{a}$ décadas da vida, sendo constituída de quadros de hemorragia subaracnóidea, síndromes deficitárias contralaterais, síndromes mesodiencefálicas, perturbações mentais as mais variadas e hidrocéfalo por hiperprodução liquórica.

Trabalho do Departamento de Cirurgia (Neurocirurgia) do Hospital Santa Isabel (Blumenau, SC) apresentado no $100^{\circ}$ Congresso da Sociedade Brasileira de Neurocirurgia (Campinas, SP — julho de 1974): * Neurocirurgião. 
As alterações radiológicas descritas por Fishgold se caracterizam por alargamento dos forames transversários na coluna cervical com alargamento do forame jugular e, mais raramente, erosão unilateral do ápice da pirâmide. Pela angiografia verifica-se malformação artério-venosa na região mesodiencefálica, geralmente unilateral e, na maioria das vezes, dependente das artérias coróideas. A classificação da doença é largamente discutida ${ }^{4}$, havendo uma, proposta por Paillas ${ }^{13}$, que a inclui entre as facomatoses. O referido autor classifica as doenças de Wyburn-Mason, Sturge-Weber e von Hippel-Lindau como displasias de tendência vascular, sendo as doenças de Bourneville e von Recklinghausen consideradas como displasias de tendência blastomatosa. Não se encontrou caráter genético na doença ${ }^{4}$.

Relataremos um caso raro pelas suas características, bem demonstrando a variedade de sintomas com que se manifesta a doença e a dificuldade em classificá-la adequadamente.

\section{OBSERVAÇAO}

O.G. (Reg. 53/73), sexo masculino, branco, com 26 anos de idade, internado no Hospital Santa Isabel, queixando-se de falta de força no hemicorpo esquerdo e epistaxis frequientes. Nasceu de parto normal e, aos 3 anos, os familiares notaram saliência venosa na pálbebra superior direita que aumentou de volume e número expandindo-se para a região frontal direita. Instalou-se engrossamento progressivo da pele das pálpebras superior e inferior bem como da região infraorbitária direita aparecendo, associadamente, pequenos nódulos duros nesta área. Um cirurgião plástico ressecou parcialmente as varicosidades do ângulo interno da órbita direita, passando a piorar após isso. Surgiram crises de falta de força no hemicorpo esquerdo que eram precedidas de saída súbita de sangue pela narina direita. Um mês antes do internamento passou a queixar-se de cefaléia de predomínio matinal, tendo apresentado 4 crises de falta de força no hemicorpo esquerdo com desvio da comissura labial para a direita. Exame físico - Bom estado geral com atrofia muscular no hemicorpo esquerdo. Discreta proptose linear com ptóse palpebral a direita; nevus facial de cor violácea, hipertrófico cobrindo as pálpebras superior e inferior direitas, região infraorbitária, tendo como limite inferior o ângulo externo da boca (Fig. 1). Distribuidos irregularmente na superficie do nevis existiam tipicos adenomas sebáceos. Varicosidades venosas eram visíveis na região frontal; macrocrania com assimetria facial e nasal devido a aumento da narina à direita com presenca de varicosidade na mucosa nasal do mesmo lado. Na região parietal mediana palpava-se uma saliência de consistência mole que se distendia à manobra de Valsalva. As veias jugulares apresentavam-se túrgidas com frêmito palpável no lado direito do pescoço. Um sopro sistólico era audivel na face lateral direita do pescoço tornando-se continuo em ambas as regiōes temporais e sobre os olhos. No precórdio era audível um sopro sistólico em todos os focos, principalmente no segundo espaço intercostal esquerdo, sem sinais de insuficiência cardíaca. Exame neurológico - Paciente psiquicamente alerta porém um pouco lento nas reacões. No hemicorpo esquerdo apresentava hemiparesia sensitivo-motora com hiporreflexia osteotendinosa e sinal de Babinski. Hemianopsia homônima esquerda com fundoscopia e aspecto retiniano normal. Exames complementares - Os estudos bioquímicos do sangue, hemograma, estudo de coagulação, pressão e exame do liquido cefalorraqueano foram normais. O eletrencefalograma mostrou sofrimento cerebral difuso com maior intensidade à direita. A radiografia simples de crânio (Fig. 2) mostrou assimetria craniana com elevação da pequena asa do esfenóide e aumento da espessura da 

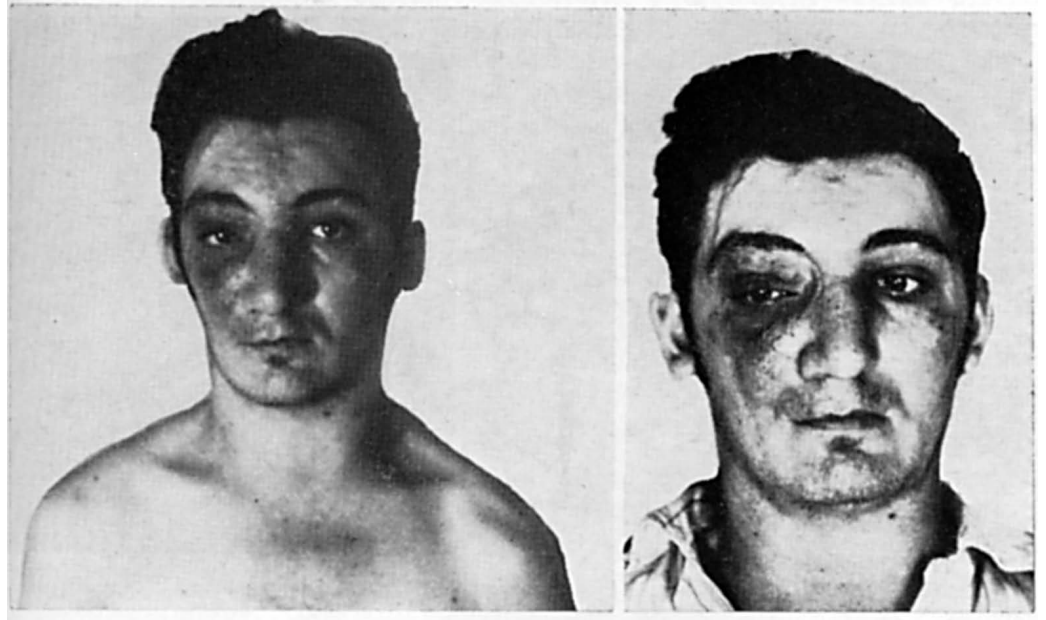

Fig. 1 - Caso O.G. A specto do paciente mostrando a macrocrania com assimetria facial. Notar o aspecto hipertrófico do angioma facial, o aumento da narina direita, os adenomas sebáceos distribuidos pelo angioma bem como as varicosidades frontais.
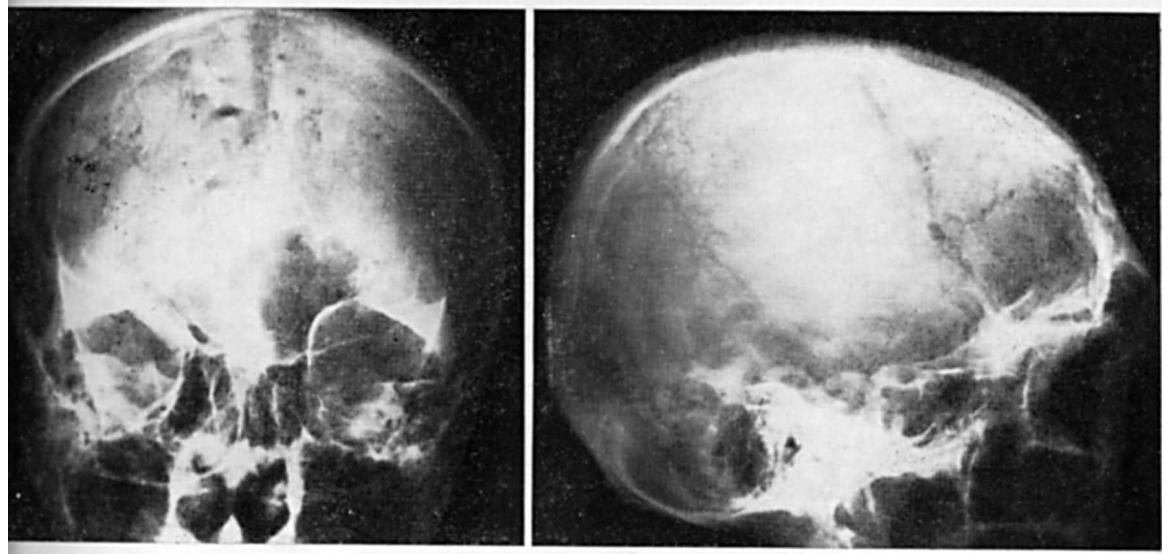

Fig. 2 - Caso O.G. Radiografias do crânio; em antero-posterior mostrando elevação da pequena asa do esfenóide, diminuição da órbita direita com presença de lagos diplóicos; em perfil observa-se calcificação da artéria basilar. 

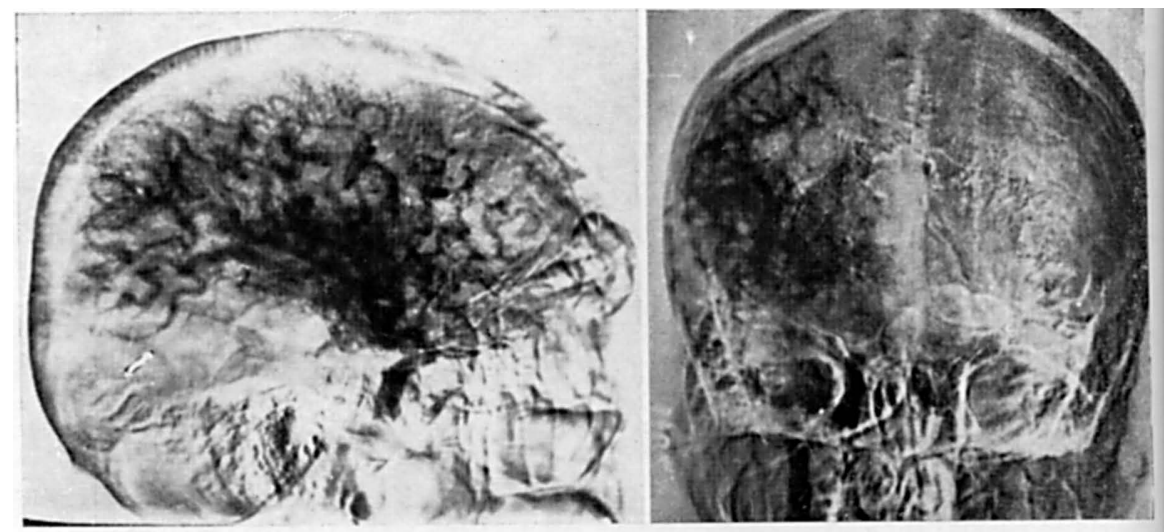

Fig. 3 - Caso O.G. Angiografia carotidea direita mostrando presença do angioma praticamente em todo o hemisfério cerebral, principalmente na região mesodiencefálica; aumento de calibre da artéria carótida interna extracraniana e da artéria oftálmica com contrastação de angioma retro-ocular.

p
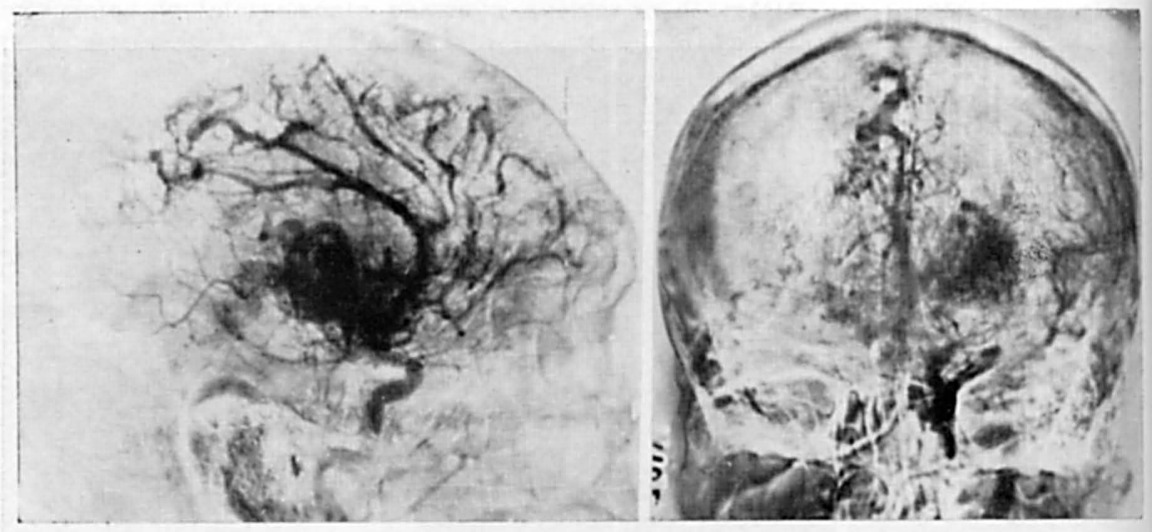

Fig. 4 - Caso O.G. Estudo angiográfico da artéria carótida esquerda demonstrando a extensão bilateral da malformação, predominantemente na região meso. diencefálica sendo nutrida por uma artéria coroidea de calibre aumentado. Note-se as telangiectasias perivasculares no trajeto da artéria pericalosa que está dilatada. Drenagem venosa precoce para veias centrais de calibre aumentado. 
calota à direita; no perfil observa-se calcificaçāo da artéria basilar. A angiografia carotídea direita (Fig. 3) mostrou dilatação do sifão carotídeo que na sua porção supraclinóidea mergulha em uma malformação angiomatosa que ocupa principalmente os núcleos da base e região diencefálica, com aumento do volume da artéria oftálmica que nutre um angioma retro-ocular. A velocidade circulatória está aumentada com inversão do sentido circulatório e drenagem venosa precoce para - seio cavernoso sem contrastação do seio longitudinal superior. O estudo da carótida esquerda (Fig. 4) mostrou extensão da malformação para o lado oposto, aumento de volume da artéria cerebral anterior com telangiectasias perivasculares no trajeto da artéria pericalosa. Nutrida por uma artéria coroidea dilatada contrastou-se uma enorme fístula artério-venosa na região dos núcleos da base e diencéfalo, com drenagem venosa precoce para as veias centrais dilatadas. Por cateterismo seletivo da artéria vertebral esquerda estudou-se o sistema vértebro-basilar que evidenciou extensão da malformação na fossa posterior especialmente no lado direito, com drenagem venosa precoce para uma veia de Galeno dilatada.
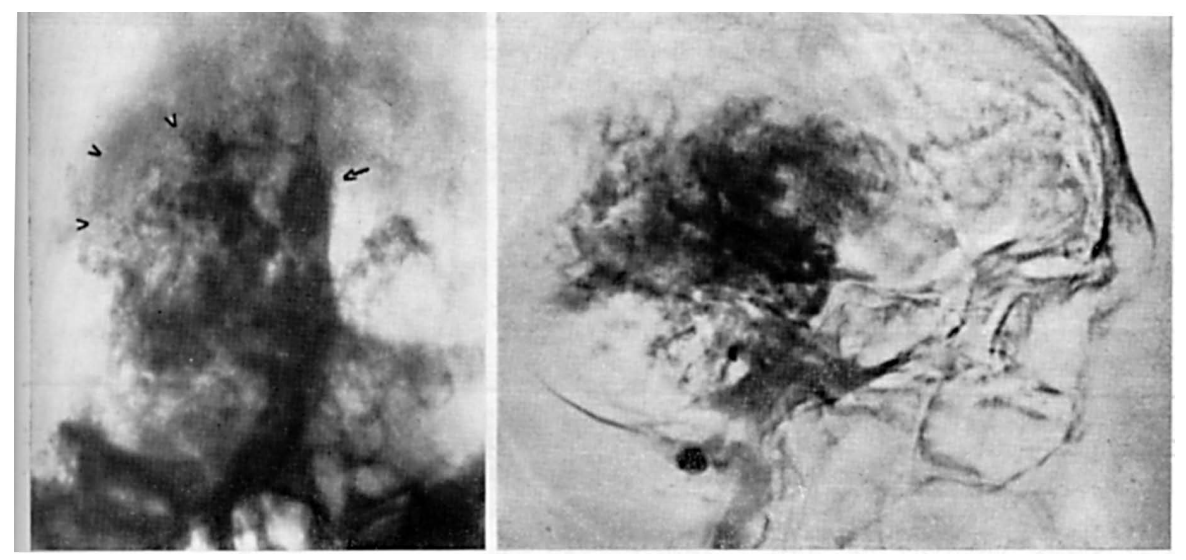

\begin{abstract}
Fig. 5-Caso O.G. Angiografia vértebro-basilar onde se evidencia a dependência da malformação do sistema vertebral. Observa-se na incidência em antero-posterior (setas pequenas) a extensão da malformação principalmente para o lado direito da fossa posterior com uma drenagem venosa precoce para uma veia de Galeno dilatada (seta maior). Na incidência de perfil observa-se dilatação da artéria vertebral ao nivel dos forames transversarios bem como aumento de volume da artéria basilar. Durante todo o estudo angiográfico tanto carotideo quanto vertebral o paciente não referiu dor à injeção do contraste.
\end{abstract}

\title{
COMENTARIOS
}

Em nenhum dos casos de síndrome de Wyburn-Mason descritos na literatura foram encontrados adenomas sebáceos associados a angiomas faciais tornando o nosso caso único, apresentando uma associação das doenças Sturge-Weber, von Hippel-Lindau e Bourneville 3, 6, 8, 9, 10, 15. Em alguns casos têm sido descritas patologias associadas como trombocitopenia e angiomas mandibulares ${ }^{12}$. 
A inversão do sentido e aumento da velocidade circulatória motivadas pelas múltiplas microfistulas geraram hipertensão ao nivel do seio cavernoso, seio lateral e veias jugulares, justificando a presença de varicosidades faciais e de couro cabeludo ${ }^{3,11}$. Esta hipertensão levou à rotura do angioma de mucosa nasal e síndrome de roubo no hemisfério cerebral direito com conseqüentes episódios de hemiparesia esquerda.

O estudo da síndrome de Wyburn-Mason bem como a revisão dos casos descritos, comparados ao presente caso, nos levou a concluir que, atualmente, não se pode classificar a doença como facomatose isolada, evidenciando a necessidade de melhores estudos.

Autores N.o de casos Angiografias

\begin{tabular}{lrr}
\hline \hline Bonnet e col. ${ }^{1}$ & 2 & \\
Wyburn-Mason ${ }^{16}$ & 22 & 4 \\
Fishgold e col. ${ }^{5}$ & 1 & 1 \\
Paillas e col. ${ }^{13}$ & 1 & 1 \\
Obrador e col. ${ }^{10}$ & 1 & 1 \\
Tasanen ${ }^{12}$ & 1 & 1 \\
Hilal e col. ${ }^{7}$ & 2 & 2 \\
Tamaki e col. ${ }^{15}$ & 1 & 1 \\
\hline
\end{tabular}

Tabela 1 - Casos de sindrome de Wyburn Mason registrados na literatura ( $\$ 1$ casos no total), sendo que apenas em 11 foi feito estudo angiográfico.

\section{RESUMO}

A sindrome de Wyburn-Mason é discutida apresentando-se uma revisão bibliográfica. Consiste na presença de angiomas anteriovenosos na face, retina e região mesodiencefálica, associada a alterações neurológicas e mentais. Um caso é apresentado, sendo comentadas suas particularidades pelas quais se evidencia que a classificação da síndrome como uma facomatose pura é discutivel merecendo melhores estudos.

\section{SUMMARY}

Wyburn Mason syndrome: a mixed phacomatosis. A case report.

The Wyburn-Mason syndrome is discussed on basis of a bibliographycal review. It consists on arteriovenous angiomas in the face, retina and mesencephalon associated with neurological and mental disturbances. A case is reported with remarks on its peculiarities. The questionability of the classification of the syndrome as an isolated phacomatosis is emphasized. 


\section{REFERENCIAS}

1. BONNET, P.; DECHAUME, J. \& BLANC, F. - L'aneurisme cirsoide de la retine (aneurisme racemeux); ses relations avec l'aneurisme de la face e l'aneurisme cirsoide de cerveau. Bull. Soc. Franc. Opthal. 51:521, 1938.

2. BROWN, D.G.; HILAL, S. K. \& TENNER, M. S. - Wyburn-Mason syndrome. Report of two cases without retinal involvement. Arch. Neurol. (Chicago) $28.67,1973$.

3. CECILE, J. P.; FOURNIER; LAINE, E.; COUSIN, J.; JOMIN, M. \& DUTHILLEUX, P. H. - Malformations arterio-veineuses cerebrales revellées par des varices peri-orbitaires chez une enfant de 3 ans. J. Radiol. Electrol. Med. Nucl. $52: 451,1971$.

4. CASTROVIEJO, P. - Diagnóstico Clinico-Radiologico en Neurologia Infantil. Editorial Cientifico-Medica, Barcelona, 1971, pág. 87-89.

5. FISHGOLD, H.; BREGEAT, Y.; LE BESNERAIS, Y. \& DAVID, M. - Inconographie de l'angiomatose neuro-retinienne (sindrome de Bonnet-Dechaume Blanc). Prèsse Médicale 60:1790, 1952.

6. FORD, F. - Diseases of the Nervous System in Infancy, Childhood and Adolecence. Charles C. Thomas, Springfield (Illinois), 1966.

7. HILAL, S.; SOLOMON, G. E.; GOLD, A. P. \& CARTER, S. - Primary cerebral arterial occlusive disease in children: neurocutaneous syndromes. Radiology 99:87, 1971.

8. KALIN, R. E. - Facial angiomatosis with angioid streats. Association of angioid streats with a component of the Sturge-Weber syndrome. Arch. Optalmol. 84:528, 1970.

9. MAY, R. \& NISSL, R. - Beitrag zur Klassifizierung der gemischten kongenitalen Angiodysplasien. Fortschrt. geb. Roentgens. Nuklearmed. 113:170-189, 1970.

10. OBRADOR, S.; LAMAS, E.; GOMES BUENO, J. \& SABORIO, F. - Um caso de angiomatosis de Bonnet Dechaume-Blanc. Revista clinica Espanhola 78: 219, 1960.

11. OBRIEN, M. S. \& SCHECHTER, M. M. - Arterio-venous malformations involving the galenic systems. Am. J. Roent. 110:50, 1970.

12. TASANEN, A. - Bonnet-Dechaume-Blanc syndrome accompanied by mandibular angiomatosis and thrombocytopenia. Brit. J. Oral. Surg. 4:213, 1967.

13. PAILLAS, J. E.; BONNAL, J. \& RIGHINI, C. - Angiome encéphalo-retino-facial (syndrome de Bonnet-Dechaume-Blanc). Revue Neurol. (Paris) 101:698, 1959.

14. RASMUSSEN, T.; MATHIESON, G. \& LE BLANC, F. - Surgical therapy of typical and a form fruste variety of the Sturge-Weber syndrome. Schw. Arch. Neurol. Neurosurg. Psychiatrie 11:393, 1972.

15. TAMAKI, N.; FUJITA \& YAMASHITA, H. - Multiple anteriovenous malformations involving yhe scalp, retina, cerebrum und posterior fossa. Case report. J. Neurosurgery 34:95, 1971.

16. WYBURN-MASON, R. - Arteriovenous aneurysm of midbrais and retina, facial naevi and mental changes. Brain 66:163, 1943.

Departamento de Cirurgia (Neurocirurgia) - Hospital Sta. Isabel - 89100 Blumenau, SC — Brasil. 